\title{
Stability of sinusoidal responses of interpolative sigma delta modulators
}

\author{
Charlotte Yuk-Fan Ho \\ Telephone: +44 (0)20 78827986 Fax: +44 (0)20 78827997 Email: charlotte.ho@elec.qmul.ac.uk \\ Department of Electronic Engineering, Queen Mary, University of London, Mile End Road, London, \\ E1 4NS, United Kingdom. \\ *Bingo Wing-Kuen Ling \\ Telephone: +44 (0)20 78482294 Fax: +44 (0)20 78482932 Email: wing-kuen.ling@kcl.ac.uk \\ Department of Electronic Engineering, Division of Engineering, King's College London, Strand, \\ London, WC2R 2LS, United Kingdom.
}

\begin{abstract}
In this paper, stability of sinusoidal responses of interpolative sigma delta modulators (SDMs) is investigated. It is found that interpolative SDMs may switch from unstable to stable behaviors even though the magnitude or the frequency of the input sinusoidal signals increase. Hence, the input magnitude stability margin and the input frequency stability margin are redefined as the minimum input magnitude and the minimum input frequency of the input sinusoidal signals such that the output of the loop filter is bounded.
\end{abstract}

\section{Introduction}

Interpolative sigma delta modulators (SDMs) are systems that consist of a negative feedback of a loop filter and a quantizer, as shown in Figure 1. The quantizer is usually very coarse and a single bit quantizer is the most common one employed in industries [1]. An input signal is oversampled and the most common oversampling ratios are 32, 64, 128 and 256 [1]. As a result, the signal band is very narrow. If the quantizer is modeled by an additive white Gaussian noise source, then the loop filter 
can be designed in such a way that the closed loop noise transfer function in the signal band is small. As a result, the quantization noise can be separated from the input signal and a very high signal-to-noise ratio can be achieved even though a very coarse quantizer is employed. This working principle refers to the noise shaping characteristics. Since interpolative SDMs can achieve very high signal-to-noise ratios, they are found in analog-to-digital and digital-to-analog conversions of many circuits and systems, particularly in audio systems [1].

It was reported in [2] that the loop filter should be designed to be an unstable one in order to achieve very high signal-to-noise ratios. However, in this case the output of the loop filter may be unbounded. For most of interpolative SDMs employed in industries, the output of the loop filter would diverge as the magnitude of sinusoidal inputs increases. Hence, the input magnitude stability margin of interpolative SDMs is usually defined as the magnitude of the sinusoidal inputs that the output of the loop filter changes from stable to unstable behaviors.

There are some fundamental issues that have not been investigated yet. For examples, does there exist a unique input magnitude, denoted as $U_{0}$, such that the output of the loop filter would change from stable to unstable behaviors? If no, then how can we define the input magnitude stability margin? Similarly, does there exist a unique input frequency, denotes as $\omega_{0}$, such that the output of the loop filter would change from stable to unstable behaviors? If no, then how can we define the input frequency stability margin? This paper is to address these issues. The outline in this paper is as follows. In Section II, our main results will be presented. Finally, a conclusion is summarized in Section III. 


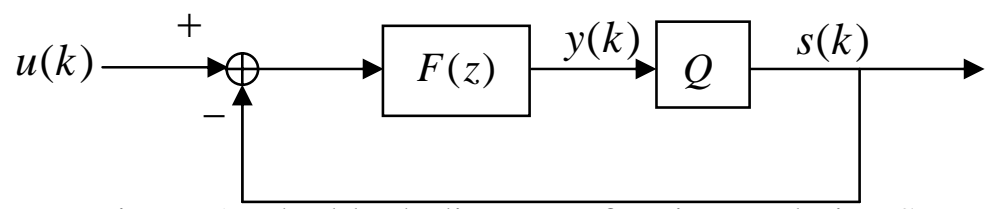

Figure 1. The block diagram of an interpolative SDM.

\section{Main results}

Denote $u(k), y(k), s(k), F(z)$ and $Q(y)$ as the input of interpolative SDMs, the output of the loop filter, the output sequence of interpolative SDMs, the transfer function of the loop filter and the quantizer, respectively, as shown in Figure 1. Assume that $F(z)$ is rational, real and strictly causal with a delay element in the numerator of the transfer function. We make these assumptions because of implementation reasons and the feedback loop configuration. Denote A, B, C and $D$ as the state space matrices of $F(z)$. Then $D=0$. We also assume that the decision boundary of a single bit quantizer is at the origin, that is $Q(y) \equiv\left\{\begin{array}{cc}1 & y \geq 0 \\ -1 & \text { otherwise }\end{array}\right.$, because this single bit quantizer is the most common one employed in industries [1]. Define $\mathbf{x}(k)$ as the state vector of the interpolative SDMs. Then the dynamics of the interpolative SDMs can be represented by the following state space equations:

$$
\begin{gathered}
\mathbf{x}(k+1)=\mathbf{A} \mathbf{x}(k)+\mathbf{B}(u(k)-s(k)), \\
y(k)=\mathbf{C x}(k),
\end{gathered}
$$

and

$$
s(k)=Q(y(k))
$$

In this paper, we only consider the case when $\mathbf{x}(0)=\mathbf{0}, u(k)=U \sin (\omega k)$, $\begin{aligned} \mathbf{A} & =\left[\begin{array}{ccccc}1 & 0 & 0 & 0 & 0 \\ 1 & 1 & -f_{1} & 0 & 0 \\ 0 & 1 & 1 & 0 & 0 \\ 0 & 0 & 1 & 1 & -f_{2} \\ 0 & 0 & 0 & 1 & 1\end{array}\right], \quad \mathbf{B}=\left[\begin{array}{l}1 \\ 0 \\ 0 \\ 0 \\ 0\end{array}\right] \text { and } \mathbf{C}=\left[\begin{array}{lllll}c_{1} & c_{2} & c_{3} & c_{4} & c_{5}\end{array}\right], \quad \text { where } \\ f_{1} & =0.0018, f_{2}=0.000685, \quad c_{1}=0.8637566182 \quad, \quad c_{2}=0.3613814738,\end{aligned}$ 
$c_{3}=0.090003709, c_{4}=0.0132091570$ and $c_{5}=0.0009083750$, in which $U$ and $\omega$ are the input magnitude and the input frequency of the sinusoidal signals, respectively. This realization, initial condition and the filter coefficients are the most common ones employed in audio systems [1]. Figure 2 to Figure 7 show the state responses and the output of the loop filter when $\omega=\frac{\pi}{96}$ and $U=0.59, U=0.591$, $U=0.592, U=0.593, U=0.594$ and $U=0.595$, respectively. It can be seen from Figure 2, Figure 4 and Figure 7 that the state responses and the output of the loop filter are bounded when $U=0.59, U=0.592$ and $U=0.595$, respectively, while it can be seen from Figure 3, Figure 5 and Figure 6 that the state responses and the output of the loop filter are unbounded when $U=0.591, U=0.593$ and $U=0.594$, respectively. Hence, there does not exist a unique input magnitude $U_{0}$ such that the output of the loop filter will change from stable to unstable behaviors.
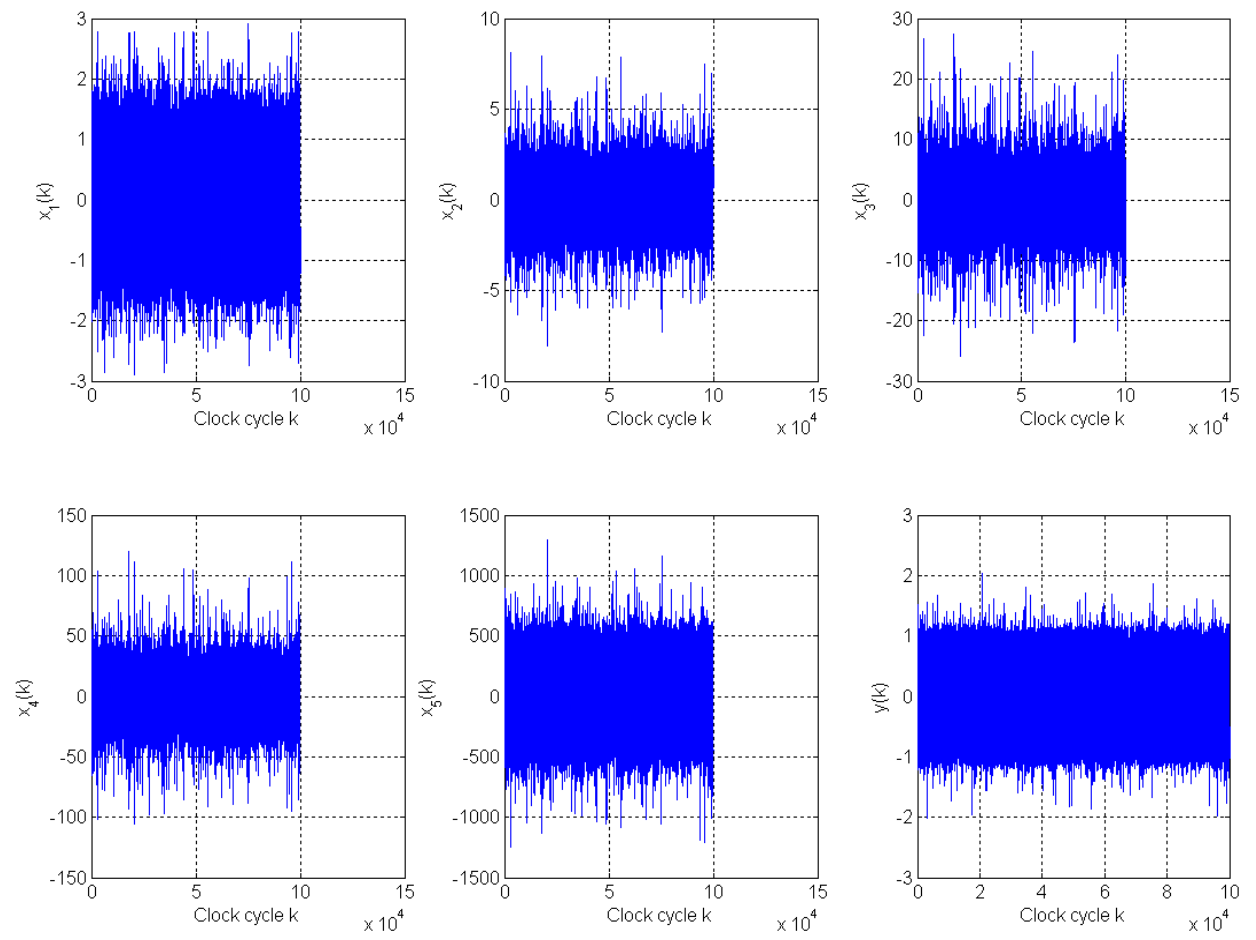

Figure 2. State responses and the output of the loop filter when $U=0.59$ and $\omega=\frac{\pi}{96}$. 

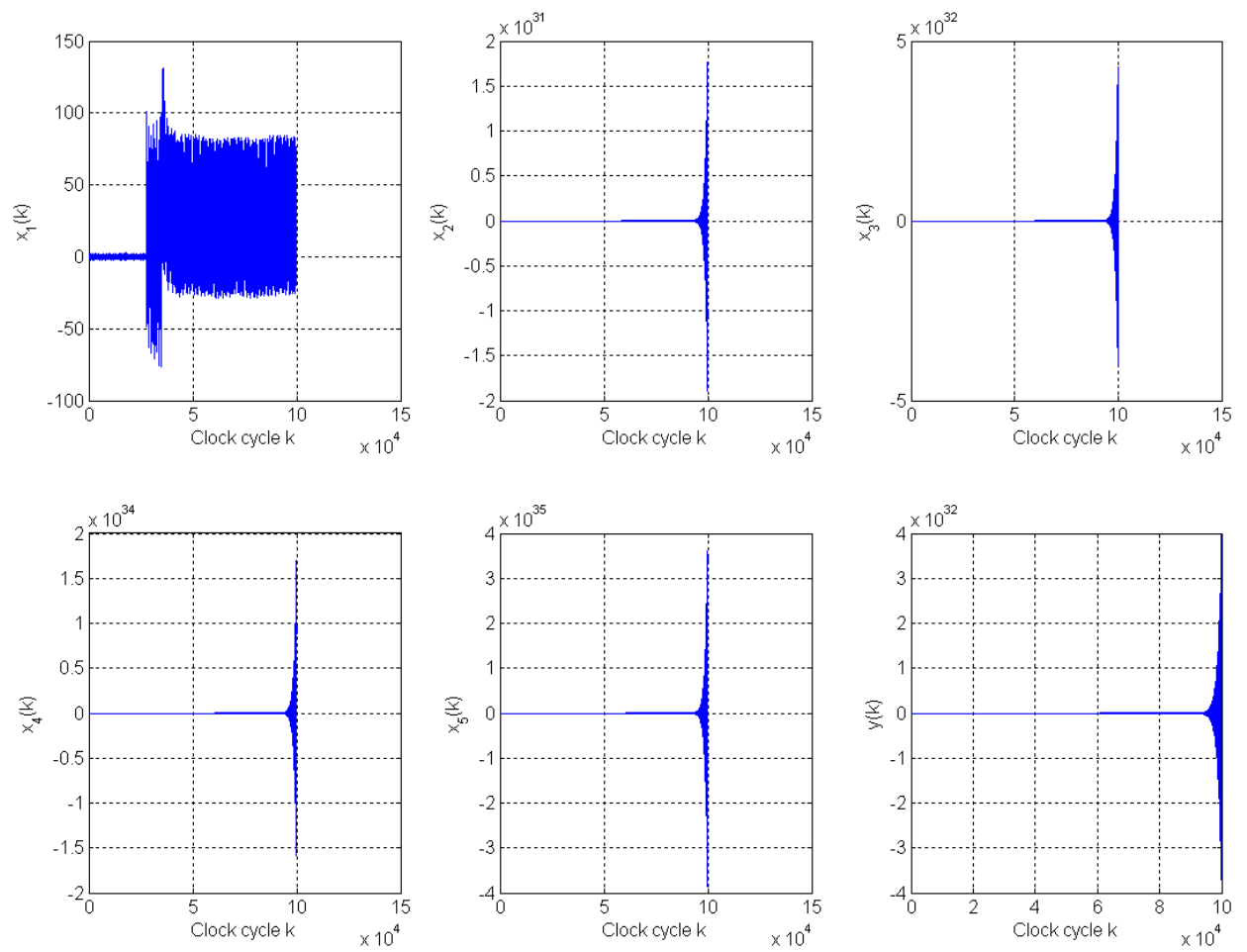

Figure 3. State responses and the output of the loop filter when $U=0.591$ and

$$
\omega=\frac{\pi}{96} \text {. }
$$
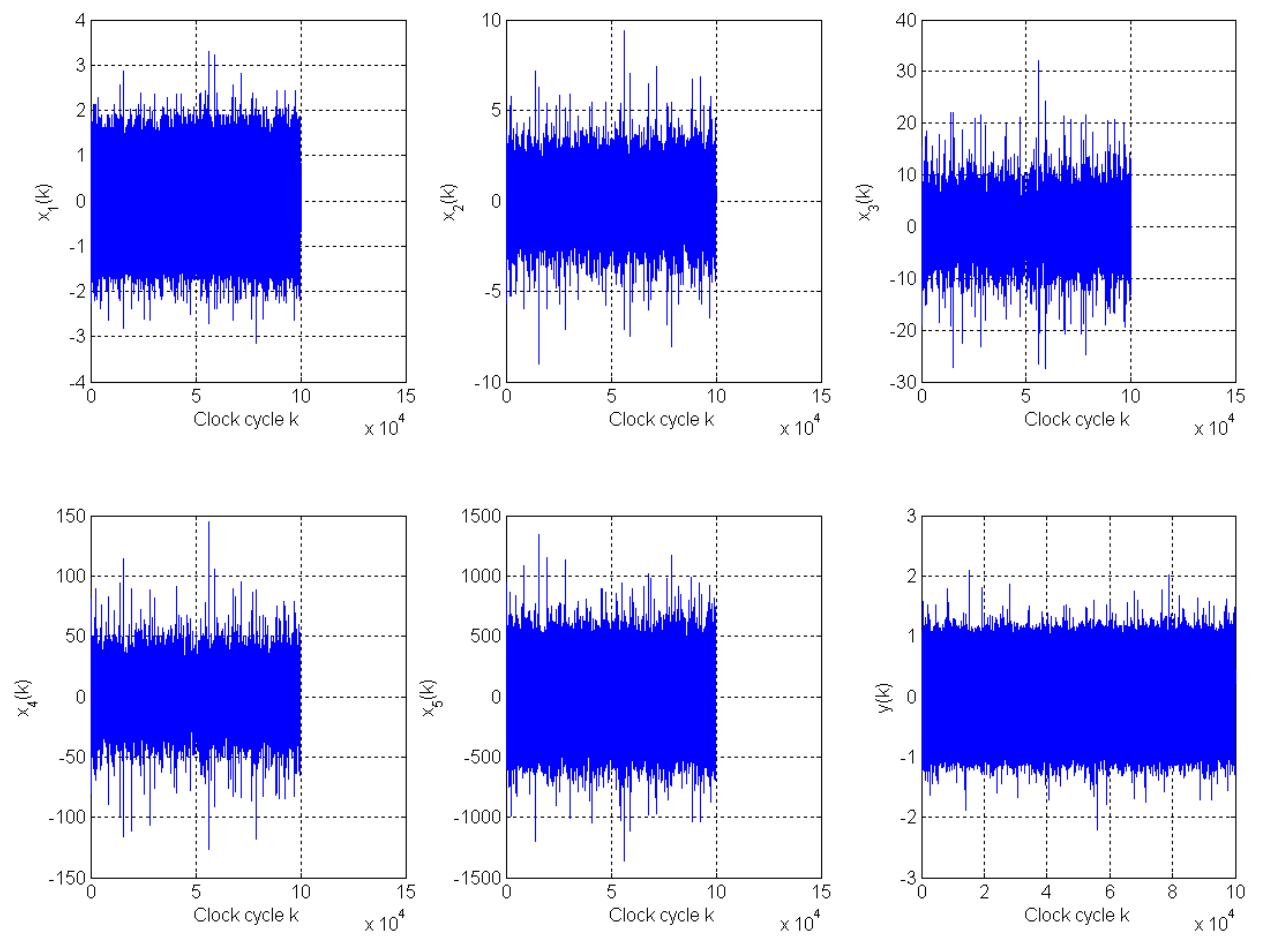

Figure 4. State responses and the output of the loop filter when $U=0.592$ and

$$
\omega=\frac{\pi}{96} .
$$



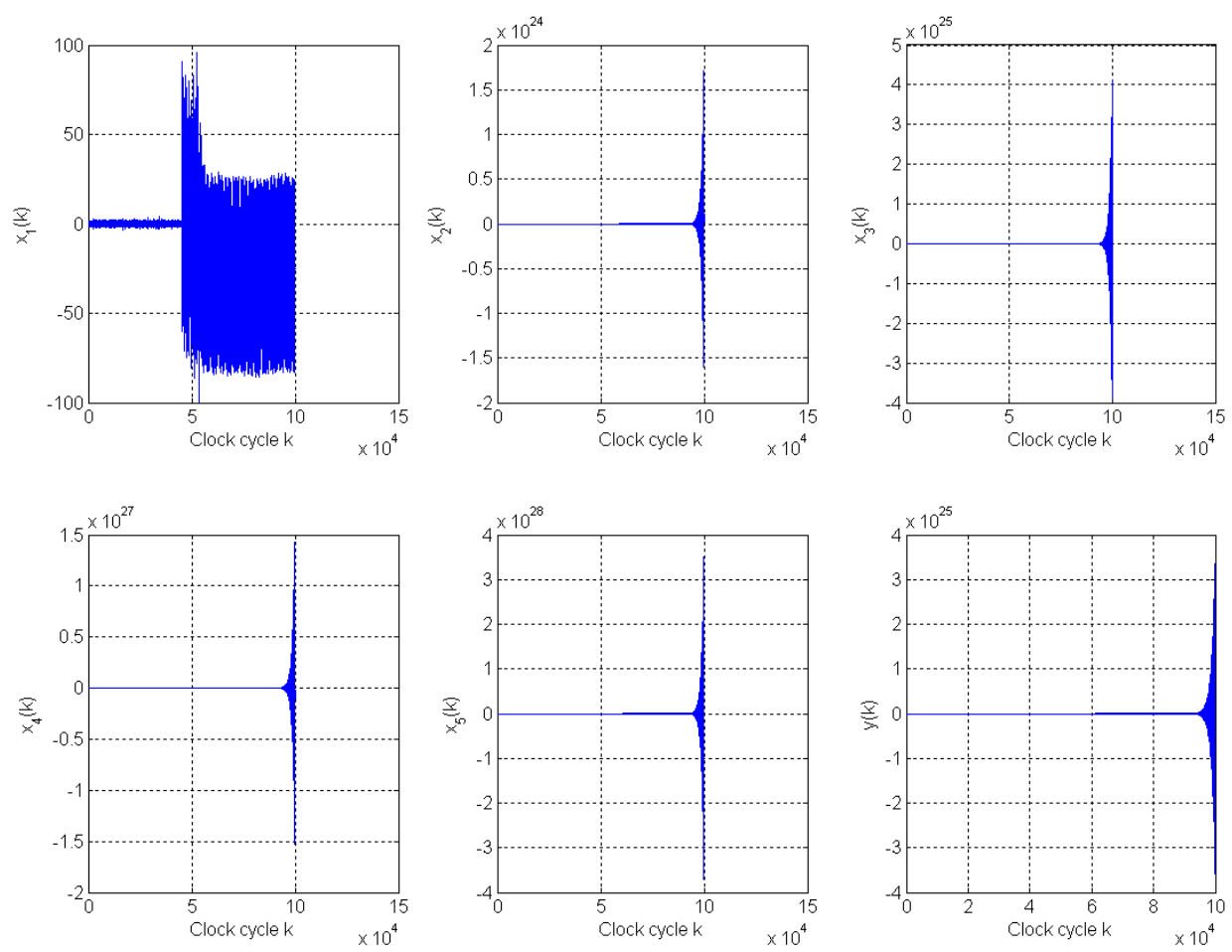

Figure 5. State responses and the output of the loop filter when $U=0.593$ and

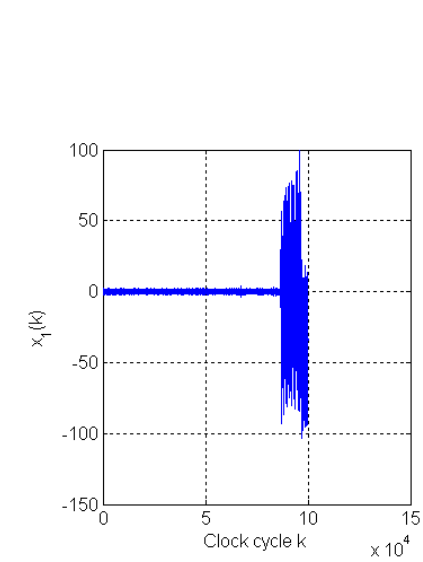

$$
\omega=\frac{\pi}{96} .
$$
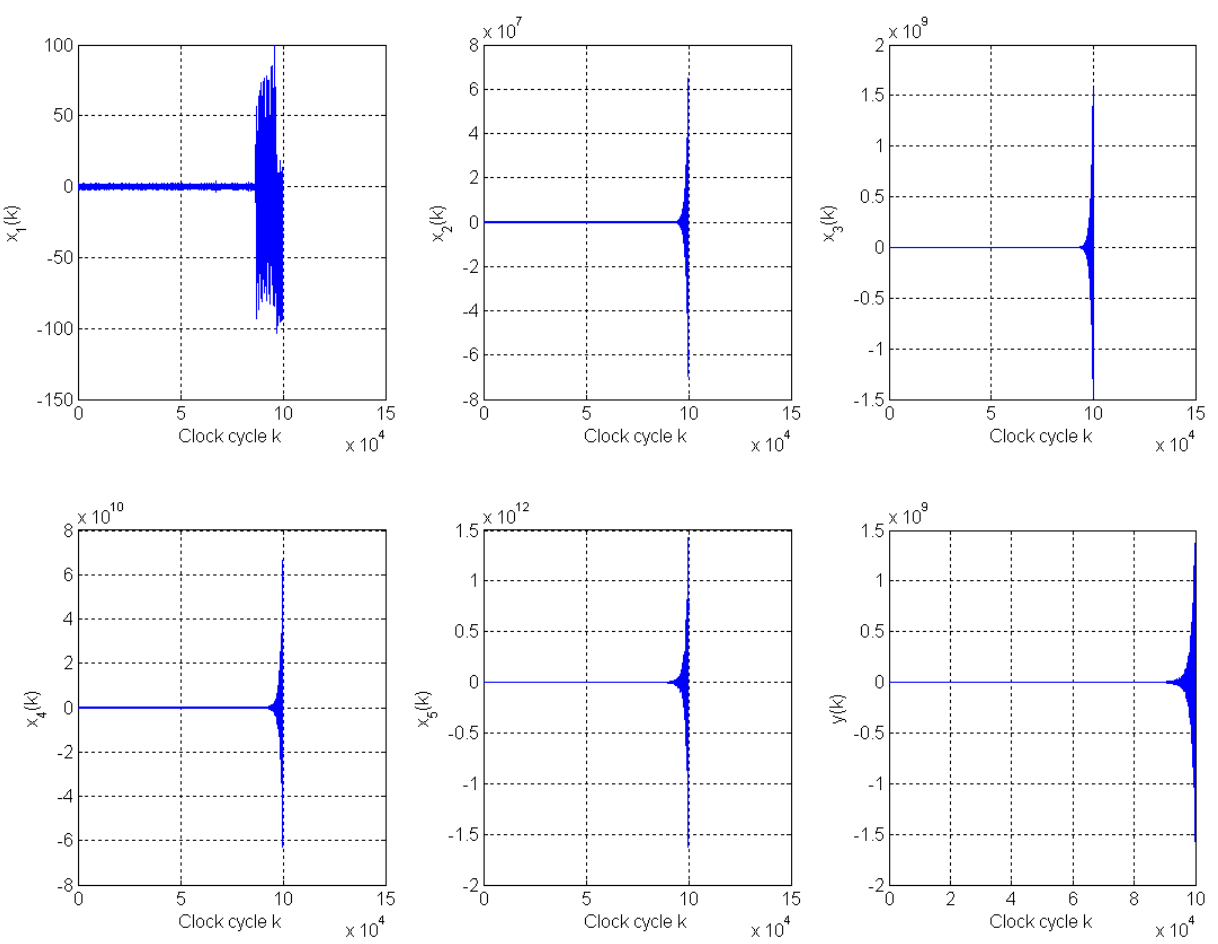

Figure 6. State responses and the output of the loop filter when $U=0.594$ and

$$
\omega=\frac{\pi}{96} .
$$



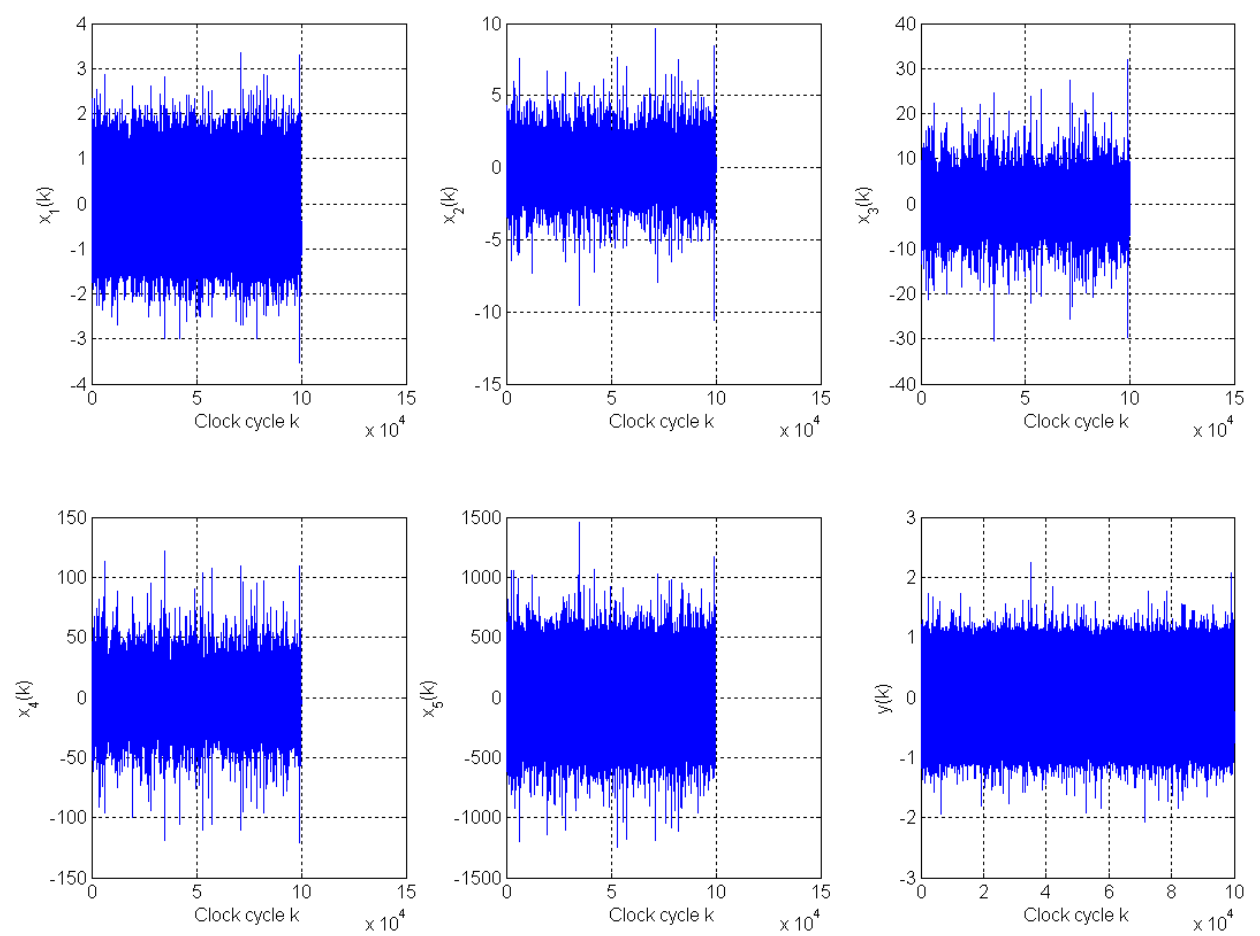

Figure 7. State responses and the output of the loop filter when $U=0.595$ and

$$
\omega=\frac{\pi}{96} .
$$

Similarly, Figure 8 and Figure 9 show the state responses and the output of the loop filter when $U=0.59$ and $\omega=\frac{\pi}{95}$ and $\omega=\frac{\pi}{97}$, respectively. It can be seen from Figure 8 and Figure 9 that the state responses and the output of the loop filter are unbounded. However, as we have shown in Figure 2 that the state responses and the output of the loop filter are bounded when $\omega=\frac{\pi}{96}$ and $U=0.59$. Hence, there does not exist a unique input frequency $\omega_{0}$ such that the output of the loop filter will change from stable to unstable behaviors. 

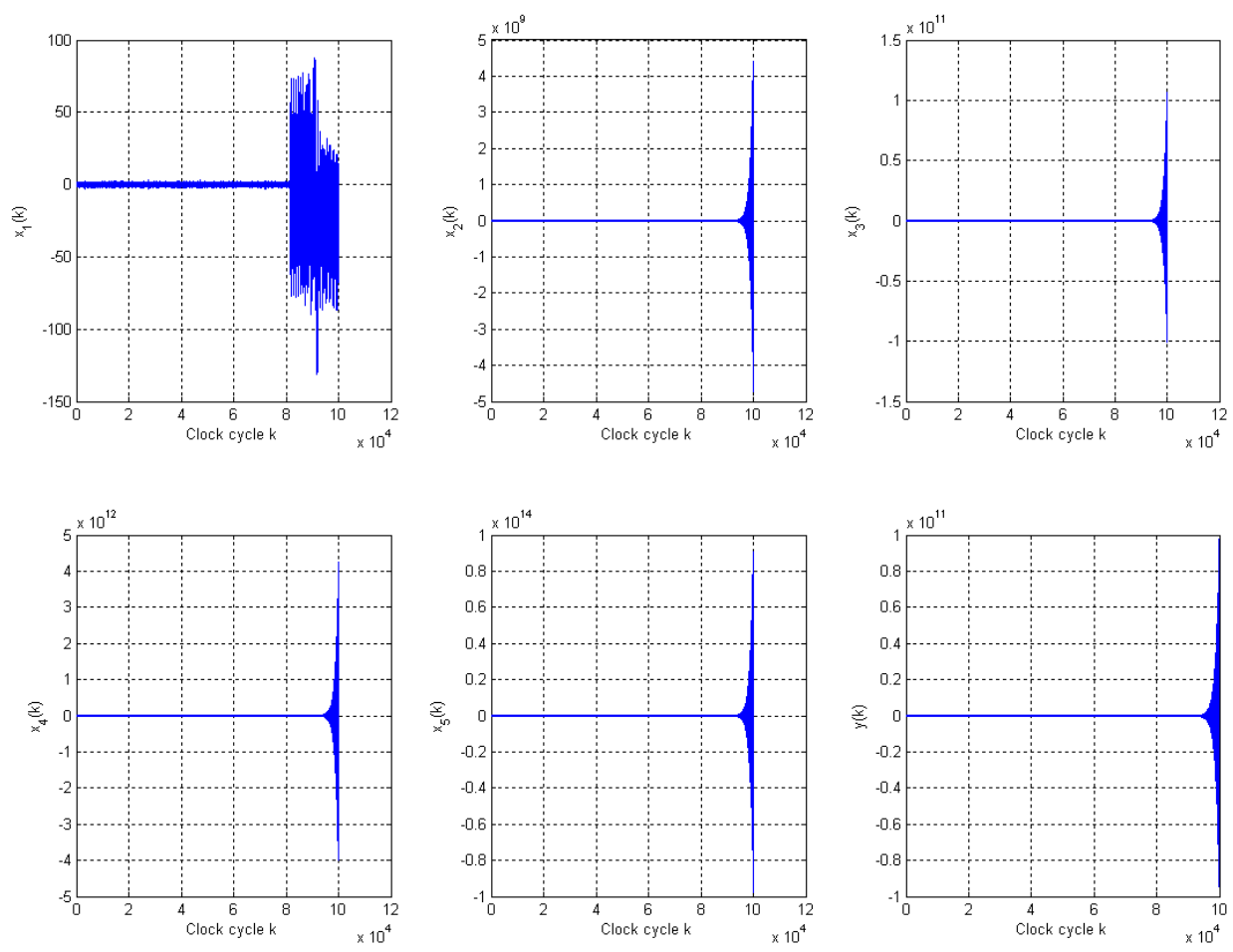

Figure 8. State responses and the output of the loop filter when $\omega=\frac{\pi}{95}$ and $U=0.59$.
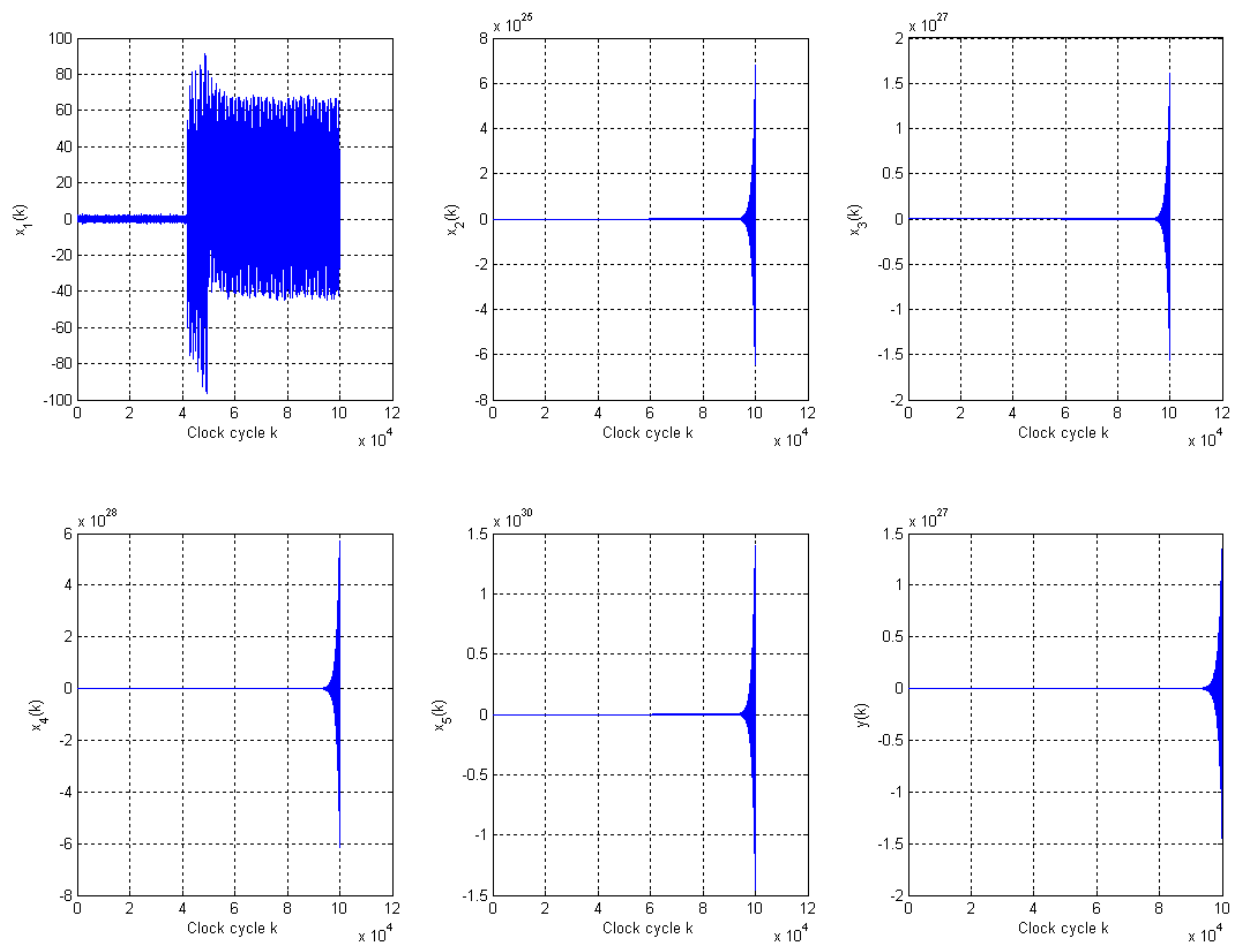

Figure 9. State responses and the output of the loop filter when $\omega=\frac{\pi}{97}$ and

$$
U=0.59 \text {. }
$$


Since there do not exist unique input magnitude and input frequency such that the output of the loop filter will change from stable to unstable behaviors, the input magnitude stability margin and the input frequency stability margin are redefined as the corresponding minimum values that the output of the loop filter is bounded.

\section{Conclusion}

In this paper, some interesting nonlinear phenomenon on the stability of sinusoidal responses of interpolative SDMs is observed. It is found that the input magnitude and the input frequency such that the output of the loop filter will change from stable to unstable behaviors are not unique. Hence, the input magnitude stability margin and the input frequency stability margin are redefined as the corresponding minimum values that the output of the loop filter is bounded.

\section{Acknowledgment}

The work obtained in this paper was supported by a research grant from Queen Mary, University of London.

\section{References}

[1]D. Reefman and E. Janssen, "Signal processing for direct stream digital: a tutorial for digital sigma delta modulation and 1-bit digital audio processing,” Philips Research, Eindhoven, White Paper, 2002.

[2]Charlotte Yuk-Fan Ho, Joshua D. Reiss and Bingo Wing-Kuen Ling, "Using SIP techniques to verify the trade-off between SNR and information capacity of a sigma delta modulator," Audio Engineering Society $120^{\text {th }}$ Convention, Paper Number 6697, 20-23 May 2006, Paris, France. 